\title{
Author Index Volume 12 (2010)
}

Abdel Hamid, H. \& BidautVeron, M. F., On the connection between two quasilinear elliptic problems with source terms of order 0 or 1

12 (2010) 727

Antontsev, S. N. \& Díaz, J. I., New $L^{1}$-gradient type estimates of solutions to one-dimensional quasilinear parabolic systems

$12(2010) 85$

Auckly, D. \& Kapitanski, L., The Pontrjagin-Hopf invariants for Sobolev maps

12 (2010) 121

Ballester-Bolinches, A., Cossey, J. \& Soler-Escrivà, X., On a permutability property of subgroups of finite soluble groups

12 (2010) 207

Benalili, M., Nodal solutions to quasilinear elliptic equations on compact Riemannian manifolds

Bidaut-Veron, M. F., see Abdel Hamid, H.

12 (2010) 909

12 (2010) 727

Bourgeois, F. \& van Koert, O., Contact homology of left-handed stabilizations and plumbing of open books

12 (2010) 223

Chabrowski, J., Peral, I. \& Ruf, B., On an eigenvalue problem involving the Hardy potential

12 (2010) 953

Chang, T., Extension and restriction theorems in anisotropic Besov spaces

12 (2010) 265

Cheng, Q.-M., see Wei, G. 12 (2010) 997

Cossey, J., see BallesterBolinches, A.
12 (2010) 207
Costa, A. \& Farber, M., Motion planning in spaces with small fundamental groups

12 (2010) 107

Degiovanni, M., Lancelotti, S. \& Perera, K., Nontrivial solutions of $p$-superlinear $p$-Laplacian problems via a cohomological local splitting

12 (2010) 475

Del Pezzo, L. M., Optimization problem for extremals of the trace inequality in domains with holes

12 (2010) 569

Deng, S. \& Hou, Z., Weakly symmetric Finsler spaces

Díaz, J. I., see Antontsev, S. $\mathrm{N}$.

12 (2010) 309

$12(2010) 85$

Dinaburg, E., Li, D. \& Sinai, Y. G., Navier-Stokes system on the flat cylinder and unit square with slip boundary conditions

12 (2010) 325

Ding, F. \& Geiges, H., Legendrian helix and cable links

dos Santos, E. M., Positive solutions for a fourth-order quasilinear equation with critical Sobolev exponent

Dou, J., Picone inequalities for $p$-sub-Laplacian on the Heisenberg group and its applications

12 (2010) 295

12 (2010) 1

Druet, O. \& Hebey, E., Existence and a priori bounds for electrostatic KleinGordon-Maxwell systems in fully inhomogeneous spaces

12 (2010) 831

Farber, M., see Costa, A. 12 (2010) 107

Filippucci, R., Pucci, P. \& Rigoli, M., Nonlinear 
weighted $p$-Laplacian elliptic inequalities with gradient terms

García-Falset, J. \& Reich, S., Integral solutions to a class of nonlocal evolution equations

Geiges, H., see Ding, F.

Ghergu, M. \& Rădulescu, V., Turing patterns in general reaction-diffusion systems of Brusselator type

Gomes, D. A., Jung, N. \& Lopes, A. O., Minimax probabilities for AubryMather problems

Gover, A. R. \& Leitner, F., A class of compact PoincaréEinstein manifolds: Properties and construction

Guo, Y., Non-existence, monotonicity for positive solutions of semilinear elliptic system in $\mathbb{R}_{+}^{n}$

Hebey, E., see Druet, O.

Höring, A., Minimal classes on the intermediate Jacobian of a generic cubic threefold

Hou, Z., see Deng, S.

Huang, H., Global error bounds with exponents for multifunctions with set constraints

Huang, J., A characterization of Hardy space on strongly Lipschitz domains of $\mathbb{R}^{n}$ by Littlewood-Paley-Stein function

Jung, N., see Gomes, D. A.

Junges Miotto, T., The Aleksandrov-BakelmanPucci estimates for singular fully nonlinear operators

Kapitanski, L., see Auckly, D.

Koshkin, S., Gauge theory of Faddeev-Skyrme functionals

Kristály, A., Mihăilescu, M., Rădulescu, V. \& Tersian, S., Spectral estimates for a nonhomogeneous difference problem
12 (2010) 607

12 (2010) 121

12 (2010) 501

12 (2010) 1031

12 (2010) 487

12 (2010) 661

12 (2010) 789

12 (2010) 629

12 (2010) 351

12 (2010) 831

12 (2010) 55

12 (2010) 309

12 (2010) 417

12 (2010) 71

12 (2010) 789

12 (2010) 871

12 (2010) 1015
Kuznetsov, A., Manivel, L. \& Markushevich, D., AbelJacobi maps for hypersurfaces and noncommutative Calabi-Yau's

12 (2010) 373

Lancelotti, S., see Degiovanni, $\mathrm{M}$.

12 (2010) 475

12 (2010) 629

12 (2010) 325

Li, D., see Dinaburg, E.

Li, G., Peng, S. \& Yan, S., Infinitely many positive solutions for the nonlinear Schrödinger-Poisson system

12 (2010) 1069

Li, H., see Wei, G.

Li, W. \& Yan, P., Continuity and continuous differentiability of half-eigenvalues in potentials

12 (2010) 977

Lopes, A. O., see Gomes, D. A.

12 (2010) 789

Lü, R. \& Zhao, K., Classification of irreducible weight modules over the twisted Heisenberg-Virasoro algebra

12 (2010) 183

Mancini, G. \& Sandeep, K., Moser-Trudinger inequality on conformal discs

12 (2010) 1055

Manivel, L., see Kuznetsov, A.

12 (2010) 373

Markushevich, D., see Kuznetsov, A.

12 (2010) 373

Mihăilescu, M., see Kristály, A.

12 (2010) 1015

Pan, S. \& Zhang, H., On a curve expanding flow with a non-local term

12 (2010) 815

Park, D., Approximating Green's functions on $\mathbb{P}^{1}$ in positive characteristic

12 (2010) 537

Peng, S., see Li, G. $\quad 12$ (2010) 1069

Peral, I., see Chabrowski, J. 12 (2010) 953

Perera, K., see Degiovanni, M.

12 (2010) 475

Peters, C., Bloch-type conjectures and an example of a three-fold of general type $\mathbf{1 2}$ (2010) 587

Pham, D. N., Groupoid Frobenius algebras

12 (2010) 939

Pucci, P., see Filippucci, R. 12 (2010) 501

Rădulescu, V., see Ghergu, M.

12 (2010) 661

Rădulescu, V., see Kristály, A.

12 (2010) 1015 
Reich, S., see García-Falset, J.

$12(2010) 1031$

Rigoli, M., see Filippucci, R. 12 (2010) 501

Ruf, B., see Chabrowski, J. 12 (2010) 953

Sandeep, K., see Mancini, G. 12 (2010) 1055

Sinai, Y. G., see Dinaburg, E.

$12(2010) 325$

Soler-Escrivà, X., see Ballester-Bolinches, A.

Stoimenow, A., Application of braiding sequences, I: On the characterization of Vassiliev and polynomial link invariants

$12(2010) 681$

Tersian, S., see Kristály, A.

Usher, M., The sharp energycapacity inequality

12 (2010) 1015

$12(2010) 457$

van Koert, O., see Bourgeois, F.

12 (2010) 223
Vétois, J., Asymptotic stablility, convexity, and Lipschitz regularity of domains in the anisotropic regime

Wei, G., Cheng, Q.-M. \& Li, H., Embedded hypersurfaces with constant $m$ th mean curvature in a unit sphere

Yan, P. \& Zhang, M., Rotation number, periodic Fučik spectrum and multiple periodic solutions

12 (2010) 437 Yan, P., see Li, W. Yan, S., see Li, G.

12 (2010) 977

12 (2010) 1069

12 (2010) 815

12 (2010) 437

Zhang, M., see Yan, P.

12 (2010) 183 